\title{
Editorial
}

\section{Modeling and Simulation in Transportation Engineering}

\author{
Wuhong Wang, ${ }^{1}$ Klaus Bengler, ${ }^{2}$ Geert Wets, ${ }^{3}$ and Huimin Niu ${ }^{4}$ \\ ${ }^{1}$ Department of Transportation Engineering, Beijing Institute of Technology, Beijing 100081, China \\ ${ }^{2}$ Department of Ergonomics, Technical University of Munich, 85747 Munich, Germany \\ ${ }^{3}$ Transportation Research Institute (IMOB), Hasselt University, 3590 Diepenbeek, Hasselt, Belgium \\ ${ }^{4}$ School of Traffic and Transportation, Lanzhou Jiaotong University, Lanzhou 730070, China
}

Correspondence should be addressed to Wuhong Wang; wangwuhong@bit.edu.cn

Received 19 November 2013; Accepted 19 November 2013

Copyright (C) 2013 Wuhong Wang et al. This is an open access article distributed under the Creative Commons Attribution License, which permits unrestricted use, distribution, and reproduction in any medium, provided the original work is properly cited.

Transportation problems including congestion, accidents and pollution emissions have become important topics, which have impact on social and economic developments. Recently, the mathematical modeling and simulation methods have received considerable attention regarding their potential as a powerful technique that can be widely applied to solve traffic and mobility operations.

This special issue presented an overview of the efforts for creating a safe, green, and intelligent transportation, especially the new achievement of intelligent transportation system (ITS) which has been reviewed in enough detail. This special issue aims at gathering the current state of the art of mathematical modeling and computational intelligence that is applied into transportation engineering. The papers submitted to this special issue have great contribution to the application of mathematical methods and models or should be the representative study in the field of transportation research. The main topics of the special issue include the following:

(i) intelligent transportation system (ITS),

(ii) logistics and transportation management,

(iii) transit and rail systems operation,

(iv) traffic flow and transportation model,

(v) mathematical optimization in traffic engineering,

(vi) traffic safety and emergence response,

(vii) renewable energy sources in transportation,

(viii) transport planning and road traffic, (ix) vehicle active safety and intelligent vehicle,

(x) energy, transport policy, and economics,

(xi) driving behavior and driver assistance system.

Since our daily life and work are closely related to traffic and mobility, transportation demand has increased dramatically. Following this increase, we additionally have to balance the increasing desire for mobility and efficiency with the societal concerns about traffic problems. Today's traffic demand is predominantly served by individual motorized vehicles, which are the primary means of transportation.

So far the significant efforts for improving transportation circumstance by using new technologies have been made. In recent years, ITS activities have focused on Green Intelligence Transportation System, which contributes to sustainability and environmental conservation compared to ITS technologies. More advanced transportation applications will materialize an accident-free traffic in the foreseeable future. Transportation engineering would no doubt move forward in the direction of safe, reliable, and economic techniques; further improvements will be made in the integration of driver assistance systems and transportation safety.

Aiming at summarizing the state-of-the-art of modeling and simulation in the context of modern transportation systems and the debate on the traffic problems, this special issue intends to introduce and discuss the developments and applications of modeling and simulation in traffic and mobility from the perspective of intelligent transportation systems. We hope that the papers in this special issue provide views of 
the research of mathematical models and the development of advanced transportation engineering applications.

Wuhong Wang

Klaus Bengler

Geert Wets

Huimin Niu 


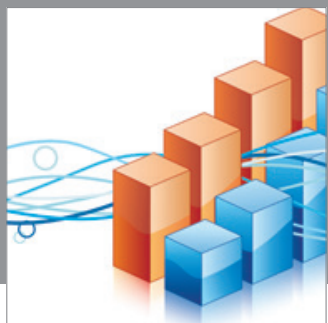

Advances in

Operations Research

mansans

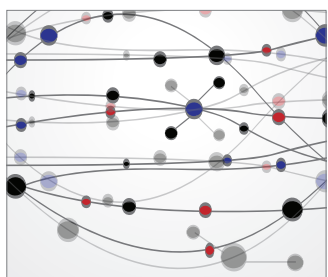

The Scientific World Journal
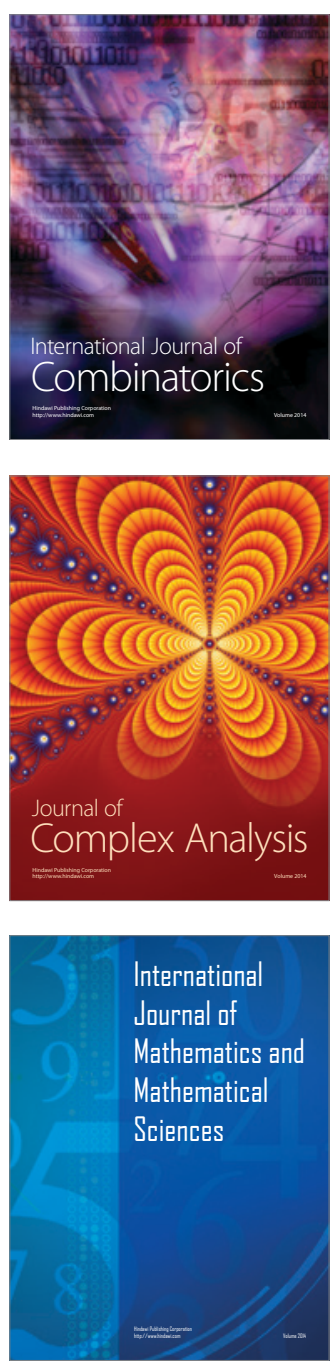
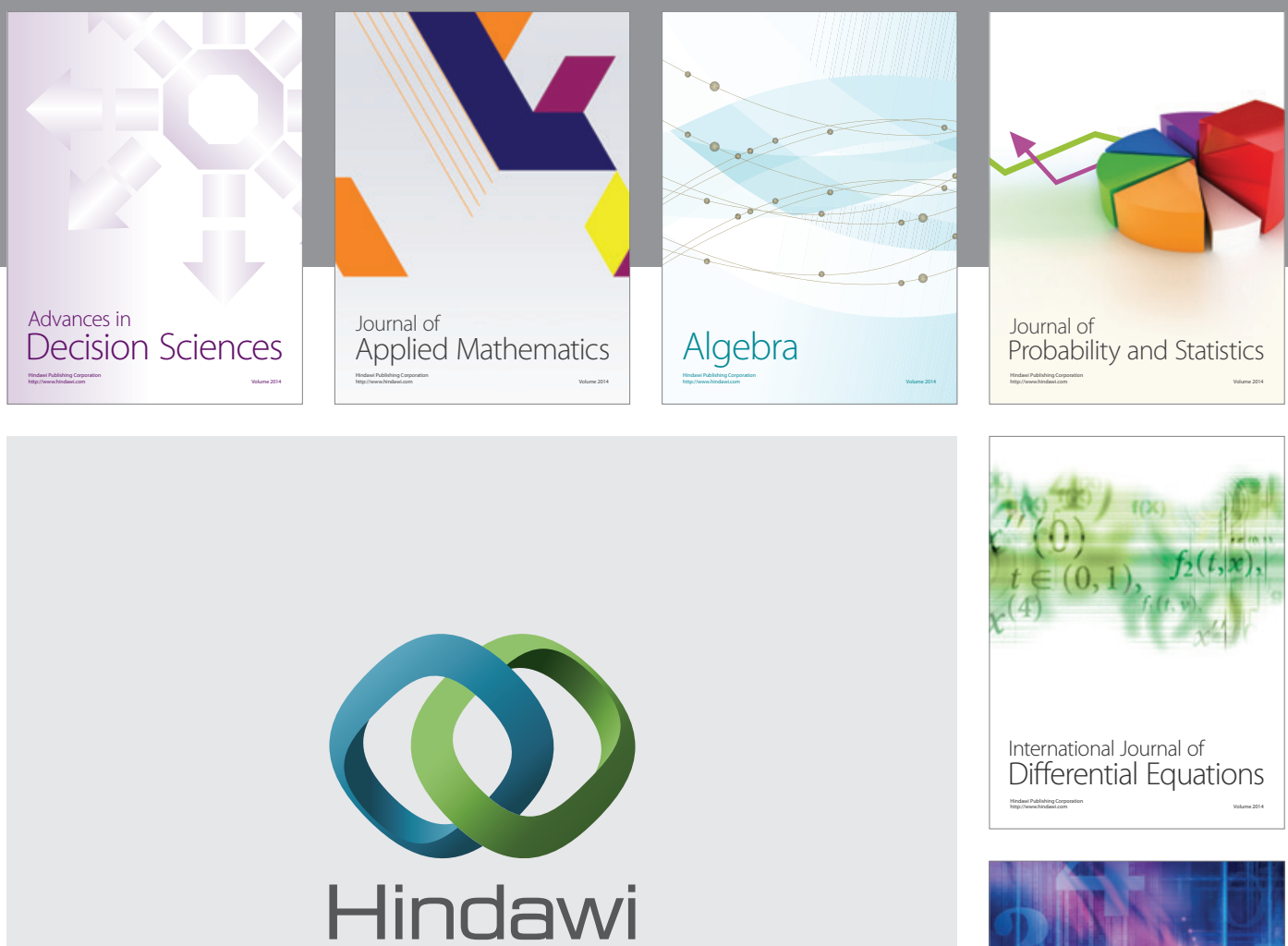

Submit your manuscripts at http://www.hindawi.com
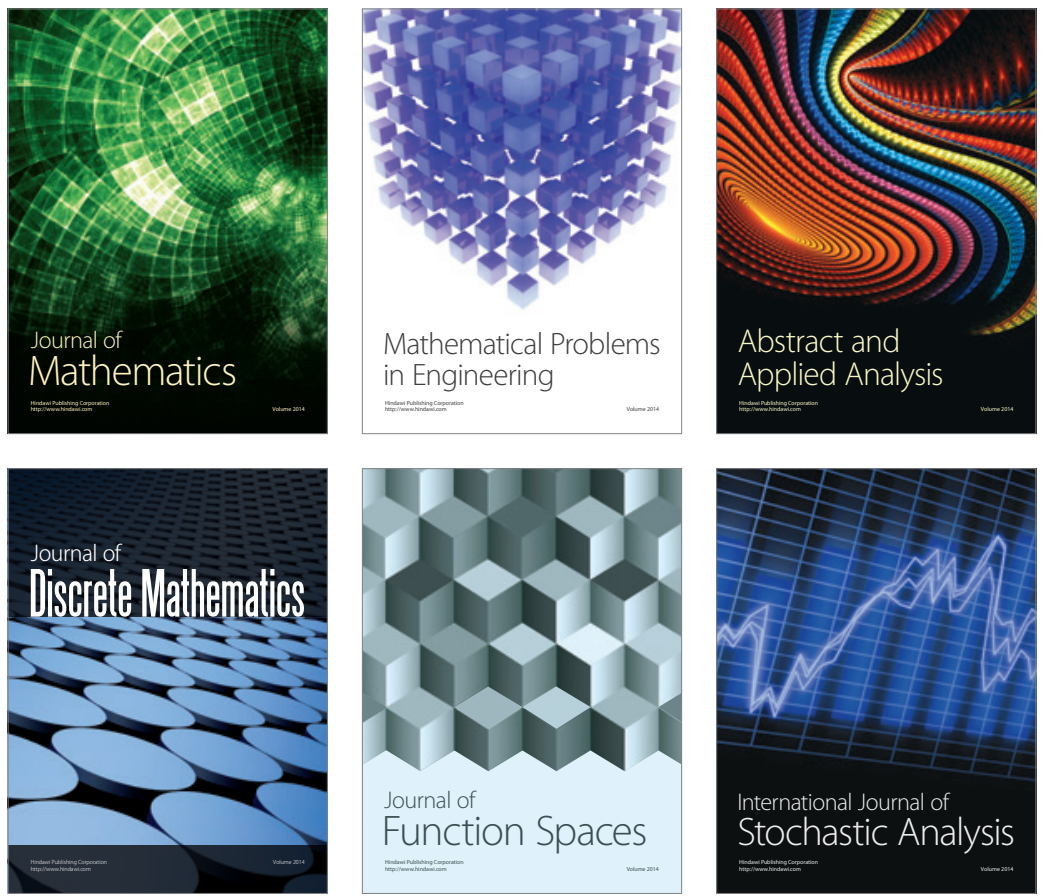

Journal of

Function Spaces

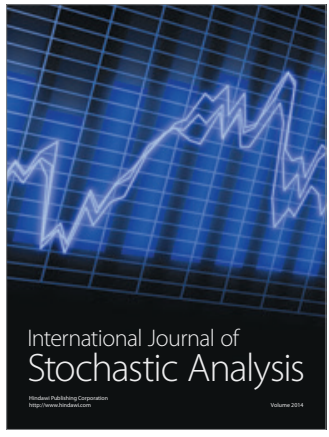

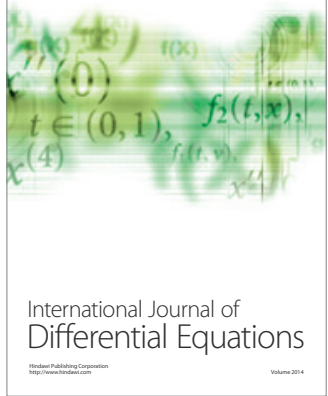
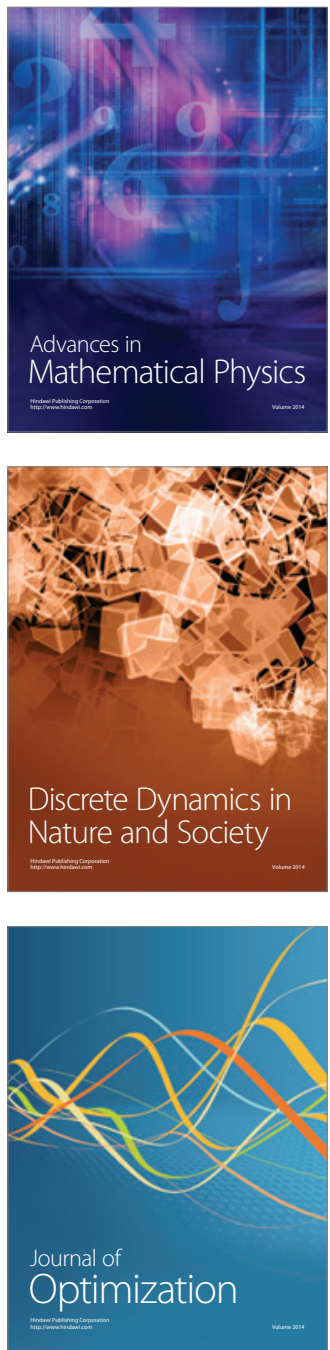